\title{
Riesgo de contaminación del acuífero freático con plaguicidas en la cuenca del arroyo Ludueña, Argentina
}

\section{Risk of contamination of phreatic aquifer with pesticides in the basin of Ludueña stream, Argentina}

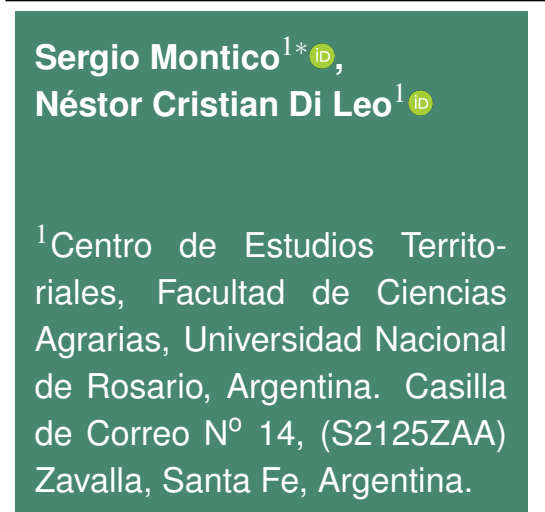

*Autor de correspondencia: smontico@unr.edu.ar

Artículo científico

Recibido: 23 de julio 2020

Aceptado: 04 de mayo 2021

Como citar: Montico S, Di Leo NC (2021) Riesgo de contaminación del acuífero freático con plaguicidas en la cuenca del arroyo Ludueña, Argentina. Ecosistemas y Recursos Agropecuarios 8(2): e2688. DOI: 10.19136/era.a8n2.2688
RESUMEN. El objetivo del trabajo fue conocer el riesgo de contaminación de acuíferos freáticos por el uso de plaguicidas en los cultivos predominantes de la cuenca del arroyo Ludueña, Santa Fe, Argentina. Se evaluaron 13 unidades cartográficas (UC) conformadas por cinco series de suelo. Se indagaron las estrategias de uso de los 14 plaguicidas más utilizados en los cultivos principales de la cuenca. El Factor de atenuación (FA) y de retardo (FR) de los plaguicidas en las series y el UC se calcularon con la aplicación de Integración de índices de riesgo ambiental (IIRAmb), también se clasificaron los resultados de FR por movilidad y FA por probabilidad de lixiviación, el valor de recarga neta de agua media con la FA de $1.2 \mathrm{~mm} \mathrm{~d}^{-1}$, y se elaboraron mapas georreferenciados del herbicida, insecticida y funguicida más móvil en el horizonte $C$ de las UC. No se halló una tendencia clara en la movilidad de los plaguicidas en los horizontes A, B y C en las cinco series de suelo. Los herbicidas fueron más móviles que los insecticidas y los fungicidas. Respecto al FA, éste fue menor en el horizonte $\mathrm{C}$, aumentando el riesgo de contaminación de los acuíferos freáticos, no se observó una diferencia clara entre los horizontes $\mathrm{A}$ y $\mathrm{B}$, con diferentes riesgos de lixiviación de plaguicidas en los diferentes ambientes de la cuenca, y el índice aplicado de retardo y atenuación de la dinámica de los plaguicidas fue útil para evaluar los riesgos de contaminación de los acuíferos freáticos.

Palabras clave: Acuíferos freáticos, cuenca rural, factor de atenuación, factor de retardo, plaguicidas.

ABSTRACT. The objective of this work was to evaluate the risk of contamination of phreatic aquifer by the use of pesticides in the main crops within the basin of the Ludueña stream, Santa Fe, Argentina. Thirteen cartographic units (CU) formed by five soil series were evaluated. Use strategies for the fourteen most used pesticides in main crops in the basin were investigated. Attenuation Factor (AF) and Retardation Factor (RF) of the pesticides in the series and $\mathrm{CU}$ were calculated by applying Integration of Environmental Risk Indices (IERI). The results of RF by mobility and AF were classified by the probability of leaching. The average net water recharge value used for the calculation of $A F$ was $1.2 \mathrm{~mm} \mathrm{~d}^{-1}$, and georeferenced maps were constructed for each most mobile herbicide, insecticide and fungicide in the $C$ horizon of the CUs. No clear trend was found in the mobility of pesticides in $A, B$ and $C$ horizons of the five soil series. Herbicides were more mobile than insecticides and fungicides. Regarding the AF, it was lower in the $C$ horizon increasing the risk of phreatic aquifer contamination, and no clear difference was observed between the horizons $A$ and B. Different risks of pesticide leaching were determined in the different environments of the basin. The applied index that considers the retardation and attenuation of the pesticide dynamics was useful to evaluate these risks.

Key words: Pesticides, basin, retardation factor, attenuation factor, phreatic aquifer. 


\section{INTRODUCCIÓN}

El nivel freático es la superficie que limita las zonas de aireación y de saturación del suelo (Varela 2014), representa un grave problema para la producción agropecuaria en condiciones climáticas con excesos hídricos, y puede convertirse en un buen recurso que aporte agua a los cultivos en períodos de déficits (Martins et al. 2019). Además del aporte de agua de las precipitaciones y el riego eventual, el drenaje profundo es una fuente importante de provisión de agua a los estratos subterráneos, y posee un rol fundamental en los puntos de recarga y descarga en las cuencas hidrográficas, influyendo en los ascensos y descensos freáticos (Zimmermann 2003). En este escenario existe una estrecha relación entre la generación de servicios hídricos de los ecosistemas (provisión de agua y regulación hidrológica) y los cambios en el uso de la tierra que en la actualidad experimenta la producción agrícola en la llanura chaco-pampeana de Argentina (Jobbágy 2011). Siendo el abandono de la rotación de cultivos anuales con pasturas y su reemplazo por esquemas de agricultura continua, sumados a la implementación de siembra directa con menor cubierta de rastrojos y presencia de malezas, lo que han llevado a un balance de agua más deficitario en toda la región (Viglizzo et al. 2009). La dinámica de los niveles freáticos expone a este recurso de agua subterránea a diferentes eventos naturales y antropogénicos que pueden alterar negativamente su calidad (Castellarín et al. 2018). En este sentido, el uso de plaguicidas para el control de malezas, insectos y hongos en las plantas cultivadas, es un riesgo (Vázquez et al. 2019). Si bien existe una tendencia a cumplir los objetivos de manejo de estas adversidades sin degradar el medio ambiente (Oerke y Dehne 2004), el desafío para el uso sostenible de la tierra es permanente (Birkhofer et al. 2015).

Los efectos asociados a la aplicación de plaguicidas en los cultivos extensivos como soja, trigo, maíz, es decir cultivos que cubren grandes extensiones que generan un producto con un relativamente bajo valor por unidad de masa, no son suficientemente monitoreados y hay dudas, intereses y demandas sociales que exigen una mayor claridad respecto a los tipos, momentos y estrategias de su uso (Montico et al. 2018). Para lograr un mejor conocimiento sobre cómo estas prácticas agronómicas podrían afectar a los distintos componentes ambientales se recurre a indicadores, los que permiten evaluarlas a través de diferentes dimensiones (Montico et al. 2013). Los indicadores utilizados para predecir el impacto y riesgo ambiental de estos productos químicos son herramientas que deberían incorporarse en las decisiones sobre la organización del uso del espacio agropecuario (Vázquez et al. 2020). Las cuales deben ser sencillas para su utilización, efectivos y fáciles de comunicar (Devilliers et al. 2005). Autores como Arregui et al. (2013) y Montico y Di Leo (2015) recurrieron en regiones de Santa Fe, a indicadores para evaluar riesgos de impacto ambiental por el uso de plaguicidas, encontrando relación entre atributos naturales y tecnologías de control de adversidades. Por lo que es importante evaluar a través de índices específicos el vínculo entre la aplicación de plaguicidas, su movilidad y potencial lixiviación hacia los acuíferos freáticos a través de los suelos (Bockstaller et al. 2008). La incorporación de propiedades edáficas al estudio de contaminación de los acuíferos freáticos por el ingreso sustancias contaminantes, modifica la valoración de su vulnerabilidad (Sainato et al. 2006, Dalpiaz y Andriulo 2017, Taboada 2018) y brinda la posibilidad de ajustar la evaluación de los impactos ambientales considerando la distribución de los suelos en una región. Por lo anterior, el objetivo del presente trabajo fue evaluar el riesgo de contaminación de los acuíferos freáticos por el uso de plaguicidas en los cultivos extensivos predominantes de una cuenca rural.

\section{MATERIALES Y MÉTODOS}

El trabajo se desarrolló en la cuenca del arroyo Ludueña (aproximadamente 80000 ha) en el sureste de la provincia de Santa Fe, Argentina, que se ubica entre los paralelos $32^{\circ} 45^{\prime}$ y $33^{\circ} 08^{\prime} S$ y los meridianos $61^{\circ} 04^{\prime}$ y $60^{\circ} 39^{\prime} \mathrm{O}$. La geomorfología dominante se corresponde con una llanura plena con muy suaves ondulaciones (pendientes máximas de 
1.2\%). El clima es subhúmedo mesotermal, con temperatura media anual de $17{ }^{\circ} \mathrm{C}$ y precipitaciones totales promedio de $1110 \mathrm{~mm}$ (Cáceres 1980), que se concentran mayormente en el período primaveroestivo-otoñal. Esta cuenca se halla en intrínseca relación con el segundo conglomerado urbano más importante de Argentina, el cual está conformado por la ciudad de Rosario y su conurbano (Figura 1). Los suelos de la cuenca se encuentran asociados a cuatro unidades de paisaje (UP), las que fueron descriptas por Bonel et al. (2005). Las UP representan una vinculación entre atributos edáficos, topográficos y de drenaje. En las UP se reconocen cinco series de suelo y sus asociaciones (Tabla 1), de las cuales sus características se reportan en la Guía de Serie de Suelos (INTA 2018), las que se distribuyen en la superficie de la cuenca caracterizada por Zimmermann (2003) y que son las unidades cartográficas (UC) en la que se evaluó el riesgo de lixiviación (Tabla 2).

En los cultivos de trigo, maíz y soya, que ocupan casi la totalidad de la superficie de uso agrícola de la cuenca y que se siembran exclusivamente en condiciones de secano (Montico et al. 2015), se indagó mediante la recopilación de información sobre la gestión del uso de herbicidas, insecticidas y fungicidas: principios activos, dosis, cantidad y oportunidad de las aplicaciones en el control de las adversidades (maleza, insectos y hongos). Para ello se recurrió a información proporcionada por cinco informantes clave, cuatro organismos oficiales, 11 publicaciones técnicas y 14 trabajos experimentales, e investigaciones regionales en sistemas productivos (Montico et al. 2014, Montico y Di Leo 2015). Con la información relevada y mediante el uso de la Integración de Índices de Riesgo Ambiental (IIRAmb) aplicado por Montico et al (2014), se calculó para la cuenca el riesgo de lixiviación a los acuíferos freáticos de los principios activos de los plaguicidas.

EI IIRAmb es una evolución del Ipest (Girardin et al. 1999), que se realiza en una hoja de cálculo que posee algoritmos y ecuaciones en entorno Excel, el cual utiliza información de su base de datos sobre los principios activos de los plaguicidas: vida media, dosis letal media $\left(\mathrm{DL}_{50}\right)$ para mamíferos, $\mathrm{DL}_{50}$ para abejas, coeficiente de adsorción en carbono orgánico (Koc), constante de Henry (Kh), coeficiente de adsorción a los coloides del suelo (Kd), ingestión diaria admisible (IDA) y toxicidad acuática, e información que se debe ingresarse sobre el clima (recarga acuífero, dirección dominante del viento). El suelo (profundidad, densidad, capacidad de campo, materia orgánica, $\mathrm{pH}$, granulometría); el sitio de aplicación (pendiente, profundidad de la napa freática, distancia a agua superficial) y la tecnología de aplicación (dosis y cobertura del cultivo). La integración de esta información en el IIRAmb se realiza mediante los índices que valoran los riesgos de impacto en aguas subterráneas: GUS (Gustafson 1989) y FAT (Rao et al. 1985); los riesgos ecotoxicológicos: RIPEST (Ferraro et al. 2003); y los módulos de riesgo para aire, agua (superficial y subterránea) y presencia del principio activos. Además, como medida de valoración total del riesgo, se calcula el impacto global -Ipest Global(Van der Werf y Zimmer 1998).

A través del IIRAmb se determinó el factor de atenuación (FA) y el de retardo (FR) de los plaguicidas mencionados, en las cinco series y en las UC. EI FA estima la fracción de pesticida que lixivia a través del perfil desde la superficie, y el FR, la capacidad que poseen para lixiviar, considerando la adsorción y distribución entre las fases sólidas y líquidas del mismo. Para optimizar la interpretación de los resultados se utilizó el FAT, el cual corresponde a la transformación logarítmica natural de FA (Bernard et al. 2005). Para la clasificación de FR y FAT se recurrió a los rangos propuestos por Khan y Liang (1989) e incorporados en el IIRAmb (Tabla 3).

Se ajustaron los tenores de materia orgánica del horizonte $A$ en todas las series de acuerdo con Bedmar et al. (2015), con el propósito de reconocer el deterioro ocasionado por el uso continuo de los suelos sometidos a agricultura por más de tres décadas. El ajuste consistió en disminuirlo un 39\% respecto a los que se indican en la Guía de Serie de Suelos (INTA 2018) y de acuerdo a lo calculado por Sainz Rozas et al. (2011), en una estimación de pérdida de materia orgánica y capacidad productiva de los suelos en la región pampeana. Las propiedades de las Series de suelo utilizadas para obtener FA y FAT 

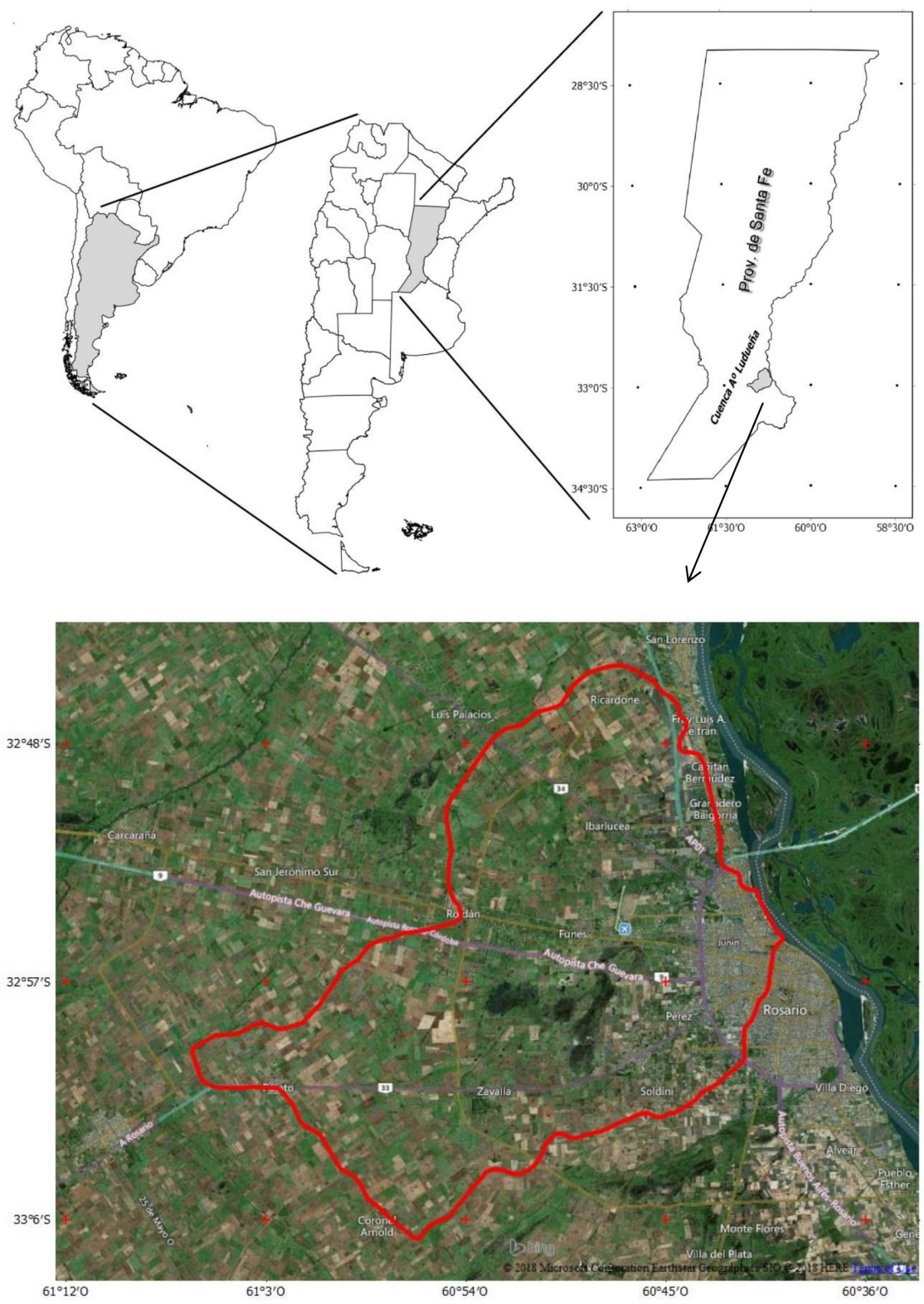

Figura 1. Cuenca del arroyo Ludueña en el sur de la provincia de Santa Fe, Argentina. Al este se halla el conglomerado urbano de la ciudad de Rosario.

Tabla 1. Características de las series de suelo presentes en la cuenca del arroyo Ludueña.

\begin{tabular}{ccccc}
\hline Serie de suelo & $\begin{array}{c}\text { Clasificación } \\
\text { taxonómica según } \\
\text { Soil Taxonomy }\end{array}$ & $\begin{array}{c}\text { Clasificación taxonómica según } \\
\text { Base referencial mundial } \\
\text { del recurso suelo (2014) }\end{array}$ & Familia & Drenaje \\
\hline Gelly & Natralbol típico & Solonetz mólico & Limosa fina, illítica, térmica & Imperfecto a pobre \\
Monte Flores & Argialbol típico & Planosol éutrico & arcillosa fina,illítica, térmica & Imperfecto \\
Peyrano & Argiudol típico & Phaeozem lúvico & arcillosa fina,llítica, térmica & Bueno \\
Roldán & Argiudol vértico & Phaeozem vértico & arcillosa fina,illítica, térmica & Bueno a moderado \\
Zavalla & Natracualf típico & Solonetz stagnico & arcillosa fina, illítica, térmica & Pobre \\
\hline
\end{tabular}


Tabla 2. Representatividad y composición porcentual de las unidades cartográficas en la cuenca del arroyo Ludueña.

\begin{tabular}{ccccccc}
\hline Unidad cartográfica (UC) & Area & Gelly & Monte Flores & Peyrano & Roldán & Zavalla \\
\hline Py & 24.7 & & & 100 & & \\
Py15 & 0.2 & & & 100 & & \\
Py20 & 1.0 & & & 100 & & \\
Py2x & 1.7 & & & 100 & & \\
Py8 & 3.0 & & & 100 & 100 & \\
Rd & 9.3 & & & & 30 & 30 \\
Rd10 & 15.3 & 20 & 20 & & 40 & \\
Rd11 & 1.8 & 20 & 40 & & 70 & \\
Rd12 & 15.9 & 10 & 20 & & 50 & 10 \\
Rd15 & 9.2 & 20 & 20 & & 70 & \\
Rd8 & 3.3 & 30 & & & & 50 \\
Za2 & 2.2 & 20 & 30 & & & 50 \\
Za3 & 8.3 & 20 & 30 & & & \\
\hline
\end{tabular}

Py: Serie Peyrano; Rd: Serie Roldán; Za: serie Zavalla. Los números de las Series indican fases, asociaciones y complejos edáficos.

Tabla 3. Clasificación de la movilidad y riesgo de los plaguicidas de acuerdo a FR y FAT (Girardin et al. 1999).

\begin{tabular}{cccc}
\hline FR & Movilidad & FAT & Riesgo \\
\hline 1 & muy móvil & $\leq 2$ & muy probable \\
$>1 \mathrm{y}<2$ & Móvil & $\geq 2 \mathrm{y}<3$ & probable \\
$\geq 2 \mathrm{y}<3$ & moderadamente móvil & $\geq 3.3 \mathrm{y}<7.2$ & moderadamente probable \\
$\geq 3 \mathrm{y}<10$ & moderadamente inmóvil & $\geq 7.2 \mathrm{y}<13.3$ & improbable \\
$\geq 10$ & muy inmóvil & $>13.3$ & muy improbable \\
\hline
\end{tabular}

FR: Factor de retardo, FAT: Factor de atenuación.

se indican en la Tabla 4. El valor de recarga neta de agua media utilizado para el cálculo de FA y FAT fue $1.2 \mathrm{~mm} \mathrm{~d}^{-1}$, según lo propuesto por Zimmermann (2003), en oportunidad del estudio de los procesos hidrológicos en la cuenca calibrando modelos de simulación en sistemas de llanura.

Mediante el sistema de información geográfica QGIS (QGIS 2017), se elaboraron mapas georreferenciados del herbicida, insecticida y funguicida más móvil en el horizonte $\mathrm{C}$ de las UC, dado representa el mayor riesgo de lixiviación por su proximidad al acuífero freático. Para ello, se calculó el FR en los horizontes y perfiles de suelo de las series y asociaciones, ponderándose la proporción que integran cada una de las UC.

\section{RESULTADOS}

La información sobre los plaguicidas en la cuenca, indica que los herbicidas de mayor uso, son: Glifosato, 2.4-D, Atrazina, Metsulfuron metil, Clorimu- ron y Dicamba; los insecticidas: Lambdacialotrina, Dimetoato y Cipermetrina; los fungicidas: Epoxiconazole, Pyraclostrobin y Azoxistrobina. Mientras que en la Tabla 5 se muestran los valores de Koc y el tiempo de vida media $\left(T^{1 / 2}\right)$ de los plaguicidas que el IIRAmb emplea para el cálculo el FAT y FR. De acuerdo al FR, no se halló una tendencia clara en la movilidad de los plaguicidas en los horizontes A, B y C en las cinco series de suelo. Los herbicidas fueron más móviles que los insecticidas y los fungicidas. Considerando el horizonte $\mathrm{C}$, los herbicidas que tuvieron mayor movilidad en orden decreciente fueron Metsulfuron metil (móvil en todas las UC), luego 2,4 D, Atrazina, Clorimuron, Metolaclor, Acetoclor y Dicamba (móviles en Py, Py15, Py20, Py2x y Py8, y moderadamente inmóvil en el resto de las UC) y glifosato (muy inmóvil en todas las UC).

El insecticida Dimetoato fue móvil en todas las UC excepto en Rd y Rd12 donde es moderadamente móvil. Los Piretroides Lambdacihalotrina y Cipermetrina, son muy inmóviles en todas las UC. Respecto a los fungicidas, Epoxiconazole es móvil 
Tabla 4. Valores de las propiedades de las series de suelo empleadas para el cálculo de FA y FAT.

\begin{tabular}{|c|c|c|c|c|c|}
\hline Serie de suelo & Horizonte & Espesor (cm) & $\begin{array}{l}\text { Densidad } \\
\text { aparente } \\
\left(\mathrm{g} \mathrm{cm}^{-3}\right)\end{array}$ & Carbono (\%) & $\begin{array}{c}\text { Humedad a capacidad } \\
\text { de campo } \\
\left(\mathrm{L} \mathrm{dd^{-1 } )}\right.\end{array}$ \\
\hline \multirow{5}{*}{ Roldán } & A1 & $0-28$ & 1.20 & 1.330 & 0.33 \\
\hline & BA & $28-40$ & 1.20 & 0.560 & 0.45 \\
\hline & Bt1 & $40-90$ & 1.25 & 0.357 & 0.45 \\
\hline & Bt2 & $90-140$ & 1.30 & 0.133 & 0.38 \\
\hline & $\mathrm{BC}$ & $140-185$ & 1.30 & 0.084 & 0.35 \\
\hline \multirow{8}{*}{ Peyrano } & $\mathrm{C}$ & +185 & 1.30 & 0.063 & 0.33 \\
\hline & A1 & $0-26$ & 1.35 & 1.540 & 0.33 \\
\hline & BA & $26-40$ & 1.35 & 1.260 & 0.35 \\
\hline & Bt1 & $40-75$ & 1.20 & 0.560 & 0.45 \\
\hline & $\mathrm{Bt} 2$ & $75-103$ & 1.25 & 0.210 & 0.45 \\
\hline & $\mathrm{BC}$ & $103-130$ & 1.35 & 0.070 & 0.38 \\
\hline & C & +160 & 1.35 & 0.070 & 0.35 \\
\hline & $\mathrm{E}$ & $0-20$ & 1.35 & 0.854 & 0.33 \\
\hline \multirow{4}{*}{ Zavalla } & Bt1 & $20-60$ & 1.28 & 0.322 & 0.33 \\
\hline & Bt2 & $60-85$ & 1.28 & 0.119 & 0.45 \\
\hline & B3 & $85-132$ & 1.28 & 0.049 & 0.45 \\
\hline & C & +132 & 1.30 & 0.056 & 0.38 \\
\hline \multirow{6}{*}{ Gelly } & A & $0-14$ & 1.35 & 0.945 & 0.33 \\
\hline & $\mathrm{E}$ & $14-24$ & 1.28 & 0.896 & 0.43 \\
\hline & Bt1 & $24-48$ & 1.28 & 0.217 & 0.43 \\
\hline & Bt2 & $48-75$ & 1.28 & 0.119 & 0.43 \\
\hline & $\mathrm{BC}$ & $75-150$ & 1.30 & 0.112 & 0.40 \\
\hline & C & +150 & 1.38 & 0.133 & 0.40 \\
\hline \multirow{6}{*}{ Monte Flores } & A1 & $0-21$ & 1.35 & 0.945 & 0.33 \\
\hline & E & $21-28$ & 1.35 & 0.910 & 0.33 \\
\hline & $\mathrm{Bt} 1$ & $28-42$ & 1.30 & 0.910 & 0.43 \\
\hline & Bt2 & $42-70$ & 1.3 & 0.945 & 0.43 \\
\hline & $B C$ & $70-135$ & 1.35 & 0.945 & 0.40 \\
\hline & C & +135 & 1.40 & 0.980 & 0.33 \\
\hline
\end{tabular}

Tabla 5. Valores de Koc y el tiempo de vida media $\left(T^{1 / 2}\right)$ de los plaguicidas evaluados.

\begin{tabular}{ccc}
\hline Pesticida & $\begin{array}{c}\text { Tiempo de vida } \\
\text { media }\left(\mathrm{T}^{1 / 2}\right)\end{array}$ & $\begin{array}{c}\text { Coeficiente de adsorción } \\
\text { en carbono (Koc) }\end{array}$ \\
\hline Glifosato & 12 & 1435 \\
$2,4-D$ & 10 & 88.4 \\
Atrazina & 75 & 100 \\
Metsulfuron metil & 10 & 32 \\
Clorimuron & 40 & 106 \\
Dicamba & 4 & 7 \\
Lambdacialotrina & 175 & 283707 \\
Dimetoato & 7.2 & 20 \\
Cipermetrina & 60 & 156250 \\
Epoxiconazole & 120 & 167 \\
Pyraclostrobin & 32 & 9304 \\
Azoxistrobina & 78 & 589 \\
\hline
\end{tabular}

en las UC Py, Py15, Py20, Py2x y Py8, y moderadamente inmóvil en el resto. Pyraclostrobina es muy inmóvil en todas la UC y Azoxistrobina es muy inmóvil en Rd, Rd10, Rd11, Rd15, Rd8, Za1 y Za2, y moderadamente móvil en Py, Py15, Py20, Py2x y Py8. En la Figura 2 se muestra la distribución espacial en la cuenca del herbicida, insecticida y fungui- cida más móviles en el horizonte $\mathrm{C}$.

Para el FAT, los menores valores se obtuvieron en el horizonte $\mathrm{C}$, y no se advirtió una diferencia clara entre los horizontes A y B (Tabla 6). Lo que indica que la mayor probabilidad de lixiviación se presenta en el horizonte profundo, y con ello el incremento del riesgo de contaminación de los acuíferos freáticos. 

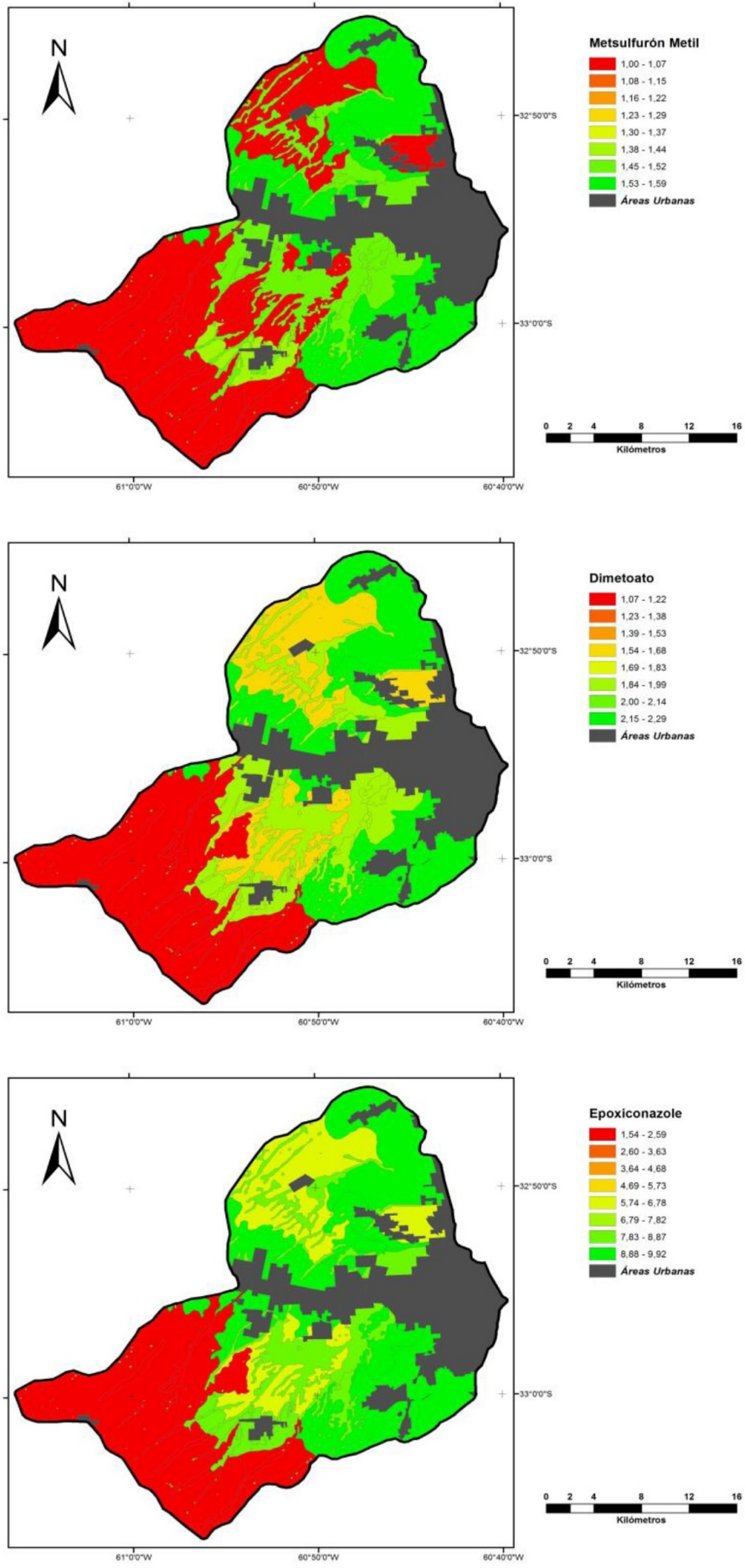

Figura 2. Representación espacial del FR (adimensional) del herbicida (Metsulfuron metil), insecticida (Dimetoato) y funguicida (Epoxiconazole) más móviles en el horizonte $\mathrm{C}$ de las UC de la cuenca. El color ojo indica los sitios de mayor riesgo, amarillo intermedio, y verde, de menor riesgo. 
Tabla 6. Probabilidad de riesgo de lixiviación a la napa de los plaguicidas para los horizontes A, B y C en la series de suelo de la cuenca.

\begin{tabular}{|c|c|c|}
\hline Plaguicida & Principio activo & Riesgo de lixiviación según horizonte \\
\hline \multirow{13}{*}{ Herbicidas } & Glifosato & En horizonte $\mathrm{C}$ de Gelly: muy probable. \\
\hline & $2,4 \mathrm{D}$ & En horizonte $\mathrm{C}$ de Gelly y Monte Flores: muy probable. \\
\hline & & En horizonte $\mathrm{C}$ de todas las series: muy probable. \\
\hline & Atrazina & Moderadamente probable y probable en los horizontes A y B de las cinco series. \\
\hline & & En horizonte $\mathrm{C}$ de todas las series: muy probable. \\
\hline & Metsulfuron metil & Moderadamente probable y probable en los horizontes A y B de las cinco series. \\
\hline & & Gelly es la serie de mayor peligrosidad potencial. \\
\hline & & En horizonte $\mathrm{C}$ de todas las series: muy probable. \\
\hline & Clorimuron & Probable y muy probable en los horizontes A y B de las cinco series. \\
\hline & Matolondr & En horizonte $\mathrm{C}$ de todas las series: muy probable. \\
\hline & Metolacior & Gelly es la serie de mayor peligrosidad potencial. \\
\hline & Acetoclor & En horizonte C de Gelly, Monte Flores y Zavalla: muy probable. \\
\hline & Dicamba & En horizonte C de Gelly, Monte Flores y Zavalla: muy probable. \\
\hline \multirow{3}{*}{ Insecticidas } & Lambdacialotrina & En horizonte C de Gelly, Monte Flores y Zavalla: muy probable. \\
\hline & Cipermetrina & En horizonte C de Gelly, Monte Flores y Zavalla: muy probable. \\
\hline & & $\begin{array}{c}\text { En horizonte C de Gelly, Monte Flores y Zavalla: muy probable. } \\
\text { Improbable en los horizontes A y B de las cinco series. }\end{array}$ \\
\hline \multirow{3}{*}{ Funguicidas } & Epoxiconazole & $\begin{array}{c}\text { En horizonte } \mathrm{C} \text { de todas las series: muy probable. } \\
\text { Moderadamente probable y probable en los hrizontes } \mathrm{A} \text { y } \mathrm{B} \text { de las cinco series }\end{array}$ \\
\hline & Pyraclostrobin & En horizonte C de Gelly, Monte Flores y Zavalla: muy probable. \\
\hline & Azoxistrobina & En horizonte $\mathrm{C}$ de Gelly, Monte Flores y Peyrano: muy probable. \\
\hline
\end{tabular}

Para los perfiles de suelo (A-B-C) de las UC, el riesgo potencial de lixiviación para los acuíferos freáticos de los plaguicidas se muestran en la Figura 3. Se presentan aquellas UC donde hubo diferencias en el FAT, con el propósito de disponer de valores diferenciales entre plaguicidas para cada UC, y con ello la posibilidad del riesgo en lixiviar. Lo que indica que para menores valores de FAT mayor es el riesgo para los acuíferos freáticos. El horizonte $C$ es el espesor de suelo que más influye en la dinámica de los plaguicidas en las UC de la cuenca, dado que mostró la mayor movilidad y probabilidad de lixiviación de principios activos desde su aplicación a los acuíferos freáticos.

El riesgo de lixiviación de todos los plaguicidas analizados fue clasificado como muy improbable en las UC (FAT>13.3), con diferencias importantes de los valores de FAT (Figura 3). Los rangos fueron: Metsulfuron metil, Metolacloro y Epoxiconazole: 17.8-37.9; Atrazina, Clorimuron y Azoxistrobina: 21.1-102.9, y Glifosato, 2,4 D, Acetoclor, Dicamba, Lambdacihalotrina, Dimetoato, Cipermetrina y Pyraclostrobina: 70.8-162.8.

\section{DISCUSIÓN}

Aunque estos plaguicidas no representan un peligro actual para la contaminación de los acuíferos freáticos de la cuenca cuando se los evaluó el IIRAmb, aquellos que poseen los valores más bajos son potencialmente riesgosos. Por lo que Metsulfuron metil, Dimetoato y Epoxiconazole, por su interacción con las características físicas y químicas de los suelos de la cuenca tienen mayor riesgo para la contaminación de los acuíferos freáticos. Además de que el riesgo podría incrementarse por cambios en el uso del suelo (proporción de agricultura/ganadería) cuestiones operativas de control de plagas (dosis de principios activos, oportunidades y cantidad de aplicaciones), procesos de degradación de suelos (pérdida de materia orgánica y erosión hídrica y eólica) y comportamiento de variables climáticas (monto y distribución de precipitaciones). En este sentido, Montico et al. (2019) afirman que, de persistir las transformaciones de los sistemas de producción y aumentar los insumos utilizados para el control de las adversidades de los cultivos, se incrementarán los riesgos de contaminación de los acuíferos. Esto en la 

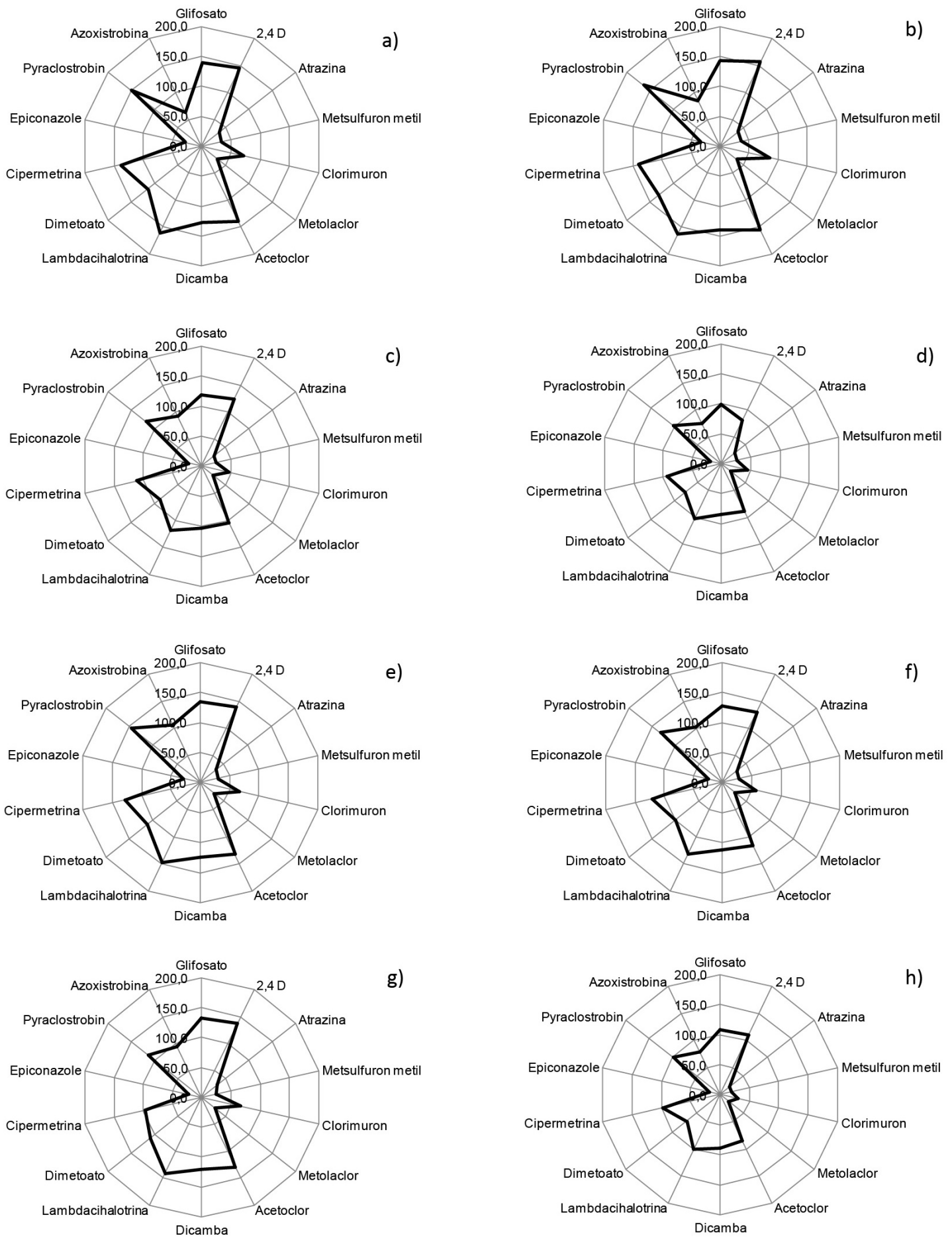

Figura 3. Valores de FAT de los plaguicidas en las unidades cartográficas en la cuenca. a) Py; b) Rd;c) Rd10; d) Rd11; e) Rd12; f) Rd15; g) Rd8; h) Za2. 
cuenca, ocurriría principalmente, en ambientes donde los suelos posean bajo tenor de coloides y se utilicen plaguicidas de elevada vida media $y$ solubilidad, con bajo coeficiente de adsorción (INTA 2015). Debido a que el FR y el FAT se obtuvieron de la base de datos del IIRAmb y no de las series estudiadas, podrían estar sub o sobre estimando los valores reales, lo que afectaría la validez de estas estimaciones. No obstante, tal como lo afirma Gallardo (2018), los modelos de simulación son herramientas que permiten obtener información de gran utilidad ante la carencia de datos reales, y posibilitan la creación y evaluación de múltiples escenarios, brindando más seguridad en la toma de decisiones. Por otra parte, estos índices no consideran el flujo preferencial de agua por los macroporos del suelo, el cual podría tener alta incidencia en el proceso de lixiviación al agua subterránea, como lo indica Aparicio et al. (2015). Al respecto, Dalpiaz y Andriulo (2017) reportan que la utilización de índices que no sólo contemplen dosis, momento y frecuencia de aplicación del pesticida, sino que incorporen propiedades edáficas, mejoran la predicción de riesgo de contaminación de las aguas subterráneas. En este sentido, y como menciona Montico (2013), también deben considerarse otras variables locales que condicionan la dinámica hidráulica-hidrológica, como las precipitaciones anuales de $1100 \mathrm{~mm}$, distribuidas en el período primavera-estivo-otoñal, la topografía subnormal con pendiente media de $0.3 \%$ y la predominancia del cultivo de soja. La aplicación de modelos de simulación que permiten conocer las interacciones entre los procesos hidrológicos en la zona saturada y en la no saturada optimizan la comprensión de la dinámica hídrica en las cuencas de llanura (Zimmermann y Riccardi 2003).

Estudios como el desarrollado en la cuenca del arroyo Ludueña, que se basan en la aplicación de modelos con biblioteca de datos, deben ser validados con información obtenida en terreno, a través del monitoreo de agua subterránea, lo que permitiría establecer la presencia de plaguicidas y su correlación con los FR y FAT calculados por estos índices. El conocimiento de las interacciones entre las prácticas agronómicas de control de adversidades y los impactos ambientales que producen, como propone Schaaf (2016), deben incorporarse a los planes de ordenamiento territoriales. La externalidades de los sistemas de producción tiene que ser internalizadas en los procesos de diseño de los modelos de uso de las tierras para alcanzar estándares de calidad ambiental compatibles con los principios de la sustentabilidad.

\section{CONCLUSIONES}

Se determinaron los riesgos de lixiviación de plaguicidas en los diferentes ambientes de la cuenca, con diferencias entre horizontes y series de suelo con respecto a la movilidad y la probabilidad de lixiviar los principios activos a los acuíferos freáticos. Los plaguicidas Metsulfuron metil, Dimetoato y Epoxiconazole, son los de mayor riesgo, condición que se presenta en el suroeste de la cuenca, en los ambientes de mejor calidad de suelos, donde se realiza una agricultura intensiva y se produce la recarga hidráulica. El horizonte $C$ es el de mayor espesor del suelo e influencia en la dinámica de los plaguicidas, por asociarse a una alta movilidad y probabilidad de lixiviación de principios activos a los acuíferos freáticos. Aunque el abordaje realizado fue satisfactorio para mejorar la interpretación de estos procesos, se requiere profundizar la evaluación con estudios locales y en ambientes reales para ajustar los resultados obtenidos. La simulación de los procesos que intervienen en el transporte de los plaguicidas de la superficie del suelo a los acuíferos freáticos, proporciona información útil para su comprensión, pero al mismo tiempo permite abrir nuevas áreas de investigación, debido a que establece focos de interés y promueve estudios específicos. La heterogeneidad espacial horizontal y vertical de los suelos debe tener consideración especial. 


\section{LITERATURA CITADA}

Aparicio V, De Gerónimo E, Hernández Guijarro K, Pérez D, Portocarrero R, Vidal C (2015) Los plaguicidas agregados al suelo y su destino en el ambiente. Ediciones INTA. Argentina. 73p.

Arregui MC, Grenón DA, Sánchez DE, Ghione J (2013) Evaluación del riesgo de impacto ambiental de plaguicidas en cultivos anuales del centro de Santa Fe. Revista Facultad de Ciencias Agrarias y Veterinarias. Sección Ciencias Agrarias 12: 13-20.

Bedmar F, Gianelli VR, Angelini H, Viglianchino L (2015) Riesgo de contaminación del agua subterránea con plaguicidas en la cuenca del arroyo El Cardalito, Argentina. Revista de Investigación Agropecuaria 41: 70-82.

Bernard H, Chabalier PF, Chopart JL, Legube B, Vauclin M (2005) Assessment of herbicide leaching risk in two tropical soils of Reunion Island (France). Journal Environmental Quality 34: 534-543.

Birkhofer K, Diehl E, Andersson J, Ekroos J, Früh-Müller A, Machnikowski F, Viktoria L, Mader VL, Nilsson L, Sasaki K, Rundlöf M, Wolters V, Smith HG (2015) Ecosystem services current challenges and opportunities for ecological research. Frontiers in Ecology and Evolution 2: Article 87. DOI: 10.3389/fevo.2014.00087.

Bonel BA, Montico S, Di Leo NC, Denoia JA, Vilche MS (2005) Análisis energético de las unidades de tierra en una cuenca rural. Revista Facultad de Ciencias Agrarias y Veterinarias, Sección Ciencias Agrarias 4: 37-47.

Cáceres LM (1980) Caracterización climática de la provincia de Santa Fe. Ministerio de Agricultura y Ganadería. Provincia de Santa Fe, Argentina. 35p.

Castellarín J, Sánchez J, Papa JC, Herrera L, Casiello F (2018) Caracterización preliminar de la problemática de calidad del agua en el área rural de Monje, provincia de Santa Fe. Energeia 15: 39-43.

Dalpiaz MJ, Andriulo A (2017) Comparación de índices de riesgo de lixiviación de plaguicidas Ciencia del Suelo 35: 365-376.

Ferraro DO, Gherza CM, Sznaider GA (2003) Evaluation of environmental impact indicators using fuzzy logic to assess the mixed cropping systems of the Inland Pampa, Argentina. Agriculture, Ecosystems and Environment 96: 1-18.

Gallardo M (2018) Revisión y análisis de estudios de modelos de cambios de usos del suelo y de escenarios a futuro. Geographicalia 70: 1-26.

Girardin P, Bockstaller C, Van der Werf H (1999) Indicators: tools to evaluate the environmental impacts of farming systems. Journal of Sustainable Agriculture 13: 5-21.

Gustafson DI (1989) Groundwater ubiquity score: a simple method for assessing pesticide leachability. Environ. Toxicology Chemical 8: 339-357.

INTA (2018) Guía de series de suelos. Estación Experimental Agropecuaria Rafaela. http://rafaela.inta.gov.ar/ mapas/suelos/_series/index.htm. Fecha de consulta: 19 de enero de 18.

Jobbágy EG (2011) Servicios hídricos de los ecosistemas y su relación con el uso de la tierra en la llanura chaco-pampeana. En: Laterra P (Ed.) Valoración de servicios ecosistémicos conceptos, herramientas y aplicaciones para el ordenamiento territorial. Ediciones INTA. Argentina. pp: 163-183.

Khan MA, Liang T (1989) Mapping pesticide contamination potential. Environmental Management 13: $233-242$. 
Martins L, Calcha J, Boero L, Yaconangelo L., Chiavassa A, Tosolini R (2019) Nivel freático en el departamento San Jerónimo (Santa Fe) Argentina. INTA EEA OLIVEROS 58: 223-230.

Montico S (2013) Vulnerabilidad de unidades de paisaje en una cuenca de Santa Fe, Argentina. Revista del CURIHAM 19:15-24.

Montico S, Denoia JA, Berardi JA (2014) Impacto y riesgo ambiental del uso de pesticidas en cultivos del arroyo Ludueña, Santa Fe. Cuadernos del CURIHAM 51: 73:79.

Montico S, Di Leo N, Bonel B, Denoia J (2018) Riesgo ambiental por el uso de fitosanitarios en cultivos anuales de la cuenca del arroyo Ludueña. Cuadernos del CURIHAM 23: 1-9.

Montico S, Di Leo N, Bonel B, Denoia J (2019) Cambios del uso de la tierra en la cuenca del arroyo Ludueña, Santa Fe: impacto en la sostenibilidad y en los servicios ecosistémicos. Cuadernos del CURIHAM 25: 31-39.

Montico S, Di Leo NC (2015) Riesgo ambiental por pesticidas en una cuenca del sur de la provincia de Santa Fe, Argentina. Revista Internacional de Contaminación Ambiental 31: 165-172.

Montico, S, Magrí L, Denoia J (2015) Impacto ambiental del uso de pesticidas en la cuenca del arroyo Ludueña Santa Fe, Argentina. Revista Ciencias Agronómicas 25: 7-17.

Oerke EC, Dehne HE (2004) Safeguarding production losses in major crops and the role of crop protection. Crop Protection 23: 275-285.

Rao PSC, Hornsby AG, Jessuep RE (1985) Indices for ranking the potential for pesticide contamination of groundwater. Soil and Crop Science Society of Florida 44: 1-8.

Sainato CM, Losinno BN, Galindo G, Landini AM, Fazio AM (2006) Geofísica aplicada a la evaluación de la vulnerabilidad a la contaminación en zonas rurales de la provincia de Buenos Aires, Argentina. Aguas Subterráneas 20: 71-82.

Sainz-Rozas H, Echeverría HE, Angelini HP (2011) Niveles de carbono orgánico y pH en suelos agrícolas de las regiones pampeana y extrapampeana argentina. Ciencia del Suelo 29: 29-37.

Schaaf AA (2016) Valoración de impacto ambiental por uso de pesticidas en la región agrícola del centro de la provincia de Santa Fe, Argentina. Revista Mexicana de Ciencias Agrícolas 7: 1237-1247.

Taboada M (2018) El suelo como recurso natural. ¿En qué marco se inserta la biorremediación? En: Brutti L, Beltrán M, García-de-Salomone I (ed.) Remediación de recursos naturales. Ediciones INTA. Argentina. pp 13-32.

Van der Werf H, Zimmer C (1998) An indicator of pesticide environmental impact based on a fuzzy expert system. Chemosphere 36: 2225-2249.

Varela R (2014) Campo de aplicación de la geología: Recursos naturales geológicos y medio ambiente. Miscelánea 21: 145-155.

Vázquez P, Sacido M, Ruiz Gonzales D (2020) Riesgos ambientales. Partido de Tres Arroyos. Región pampeana austral. Argentina. RA'e GA 47: 49-66.

Vázquez P, Zulaica L, Somoza A (2019) Agriculturización, impactos ambientales y zonificación ecológica en el partido de Tres Arroyos (provincia de Buenos Aires, Argentina). Período 2002-2017. Geografia e Ordenamento do Território 18: 209-232.

Viglizzo EF, Jobbágy EG, Carreño L, Frank FC, Aragón R, De Oro L, Salvador V (2009) The dynamics of cultivation and floods in arable lands of Central Argentina. Hydrology and Earth System Sciences 13: 491-502. 
Zimmermann ED (2003) Modelo hidrológico superficial y subterráneo desarrollado para la simulación de sistemas de llanura (II). Estrategias para la calibración. Boletín Geológico y Minero 114: 159-169.

Zimmermann ED, Riccardi G (2003) Modelo hidrológico superficial y subterráneo desarrollado para la simulación de sistemas de llanura (I).Aplicación en el sistema Ludueña (Snata Fe, Argentina). Boletín Geológico y Minero114: 147-158 